\title{
Case Study: Retrofitted Solution to AdDress Sound Flanking Via Window Mullions of StaCKed Residences
}

\author{
Pier-Gui Lalonde ${ }^{* 1}$ \\ ${ }^{1}$ Integral DX Engineering Limited, Ottawa, Ontario, Canada
}

\section{Introduction}

The subject of this case study is a sound transmission issue between stacked residences in a condominium building. The occupants of a third floor suite reported that airborne sounds originating in the suite directly above were clearly audible and disturbing within some of the rooms in their suite. The offending sounds included conversations, television loudspeakers, and the operation of window blinds. The façade of the building featured continuous window mullions of aluminium construction with hollow cores, spanning from windows on the third floor to those on the fourth. These were identified as the probable cause of the noise issue. Without consulting an acoustical engineer, the developers attempted to block a small portion of the inside of some of the mullions with a low-density spray foam product. The third floor occupants reported that this resulted in no audible improvement.

This paper will outline the approach taken to assess the sound transmission issue, and to develop mitigation that was both practical to implement and effective in improving sound transmission loss between the suites.

\section{Initial Assessment}

An objective evaluation was required to assess the extent and nature of noise transmission between the suites. Integral DX Engineering completed airborne noise isolation testing [1] between two pairs of rooms in each of the identified suites, with the noise sources located in the lower suite. Subjective observations confirmed that most of the test noise in the receiver rooms was emanating from window components. The resulting Noise Isolation Class (NIC) $[1,2]$ ratings were 53 and 54, which was well below expectations given the separating construction: concrete slab $225 \mathrm{~mm}$ thick, with floating hardwood floors above. The one-third-octave band Noise Reduction (NR) results showed that the NIC scores were limited by the performance in the $800 \mathrm{~Hz}$ and $1000 \mathrm{~Hz}$ bands. Based on a review of test data collected for similar floor-ceiling constructions elsewhere, the NR performance in those bands was approximately $20 \mathrm{~dB}$ below expectations.

Therefore, the initial assessment confirmed that the window mullions created significant sound flanking paths, bypassing the direct path through the floor-ceiling. While the overall sound isolation performance still complied with Ontario Building Code requirements between adjacent suites [3, Section 90.11.], the performance was far below the standard set for the project and which could reasonably be expected by purchasers.

\footnotetext{
*pier-gui@ integraldxengineering.ca
}

\section{Evaluation of Noise Mitigation Options}

Two primary sound transmission paths via the window mullions were identified: (1) airborne sound transmission via the hollow cores, and (2) vibration transmission along the aluminium walls, re-radiating as structure-borne sound. The relative contribution of each sound transmission path was unknown. However, both paths were considered when evaluating potential noise mitigation options. There were also practical limitations to consider for any proposed solution. Solutions which involved breaking the vibration transmission path were ruled-out, as this would involve significant construction, cost, changes to the building envelope, and changes to the look of the new building.

It is common to fill hollow window mullions or door frames with a dense material (e.g. sand or grout) to mitigate sound transmission via these components [4, p.196]. This would have provided significant mitigation of the airborne sound transmission path, as well as damping of vibrations along the aluminium walls. The application of closed-cell spray insulation to block the mullion cavities at the level of the slab was also considered. Ultimately, there were several concerns with any proposal to introduce material inside the window mullions. Holes would have had to have been drilled to access the cavities. The heat transfer characteristics of the mullions, which form part of the buiding envelope, would have changed, and there were concerns that this would increase the risk of condensation and mould growth. These concerns were compounded by the fact that a central humidifier was planned for the make up air system for the building. Finally, the mullions may not have been adequately blocked to hold aggregate materials in place.

It was proposed to instead investigate mitigation that could be applied to the suite-facing surfaces of the mullions. In addition to improving sound transmission loss, the solution would ideally need to be fully reversible. While applying treatment to mullions on both the third and fourth floors would have been preferable to maximize the potential improvement, all work needed to be done in the third-floor suite only. The appearance of the treated mullions from within the third floor suite was also a concern.

\section{Validation of Noise Mitigation}

Validation testing was completed in order to gauge the effect of applying a mass-loaded barrier to the surfaces of the window mullions. A $3 \mathrm{~m}$ long section of comparable window mullion was used for this purpose, placed horizontally on supports. Initial measurements were completed by tapping one of the suite-facing surfaces of the mullion with a wood dowel, and measuring the vibration acceleration response 
on the same surface at multiple points away from the stimulus. This testing confirmed that vibration transmission was efficient along the length of the un-treated mullion, with no change in the acceleration response at various distances away from the stimulus.

Next, the testing was repeated with approximately half the length of the mullion filled with densely-packed mineral fibre insulation. The acceleration response was reduced by approximately a factor of 2 , with the stimulus and measurement near the opposing ends of the mullion.

Finally, the insulation was removed, and tests were completed with one and two of the mullion surfaces covered with a barium-loaded vinyl product, which had been selected for a high surface weight $\left(2.0 \mathrm{lb} / \mathrm{ft}^{2}\right.$, or approximately 9.8 $\mathrm{kg} / \mathrm{m}^{2}$ ). The stimulus and measurements were both located on surfaces without the mass-loaded barrier treatment. The acceleration response was reduced by approximately a factor of 2 with two of the three suite-facing surfaces treated, compared to the un-treated condition.

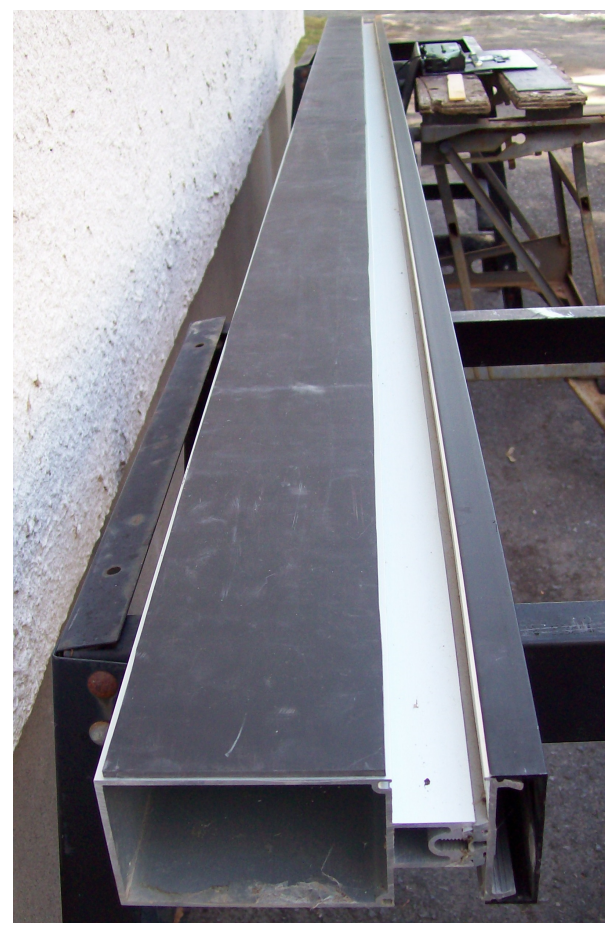

Figure 1: Validation testing setup, with mass-loaded barrier applied to one of the window mullion surfaces.

The testing results showed measurable but modest reductions of vibration transmission by adding mass-loaded barriers under the test conditions. Various adhesives were also tested for their suitability to hold the mass-loaded barrier in place and ease of removal.

\section{Final Noise Mitigation Design}

The final noise mitigation plan was to affix a massloaded barrier to each of the three suite-facing surfaces of each vertical window mullion within the third floor suite. The mass-loaded barriers would then be covered with custom-fabricated aluminium caps, colour-matched to the window frames. To ensure a more consistent look, some horizontal window mullions also received the same treatment. Compared to the validation testing, the mass-loaded barriers would cover a shorter span, but each of the three suite-facing surfaces would receive the treatment (compared to only two of three for the testing). The aluminium caps would provide additional mass, which could only further reduce airborne and struture-borne sound transfer.

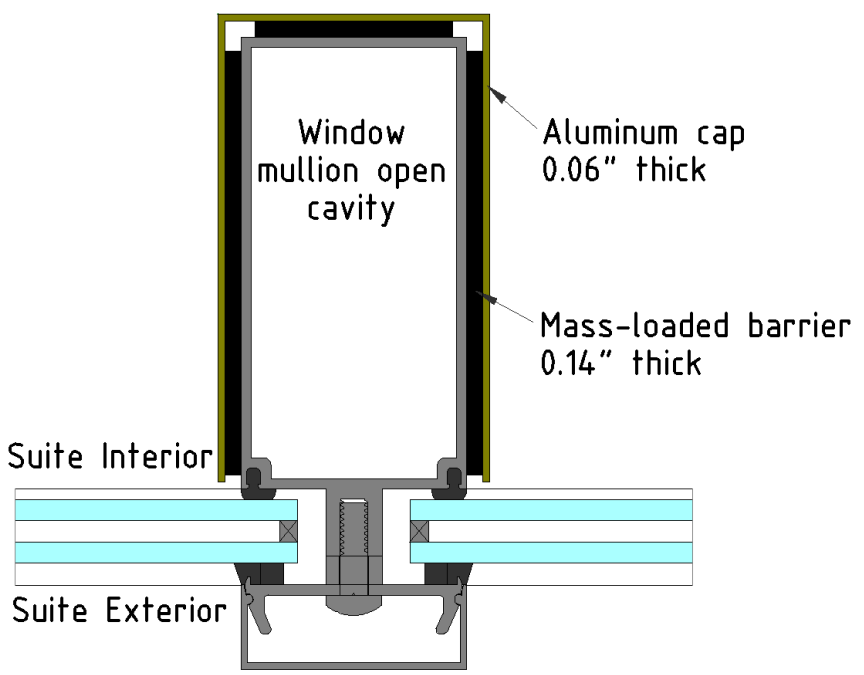

Figure 2: Cross-section of window mullion with acoustic treatment.

\section{Implementation and Results}

The mass-loaded barriers and aluminium caps were carefully prepared and installed within the third floor suite. The occupants confirmed that, subjectively, the installed noise mitigation had significantly reduced the audibility of airborne sounds from the fourth floor unit, and they were quite pleased with this result. As such, there was no clear value-added in completing any follow-up testing. However, there are other vertically-adjacent suites in the building with the same condition, and so there was a reasonable possibility that implementation of this solution would be desired elsewhere in the future. It was recommended to the Condominium Corporation that, should this be the case, objective testing of the installed mitigation should be done first.

\section{Acknowledgments}

The author would like to recognize the contributions of Gregory Clunis to the success of the project.

\section{References}

[1] ASTM E336-17a, Standard Test Method for Measurement of Airborne Sound Attenuation between Rooms in Buildings, ASTM International, West Conshohocken, PA, USA, 2017.

[2] ASTM E413-16, Classification for Rating Sound Insulation, ASTM International, West Conshohocken, PA, USA, 2016.

[3] Ontario Ministry of Municipal Affairs and Housing. Ontario Building Code 2012.

[4] M. David Egan. Architectural Acoustics. J. Ross Publishing, Inc., 5765 N. Andrews Way, Fort Lauderdale, FL 33309, 2007. 